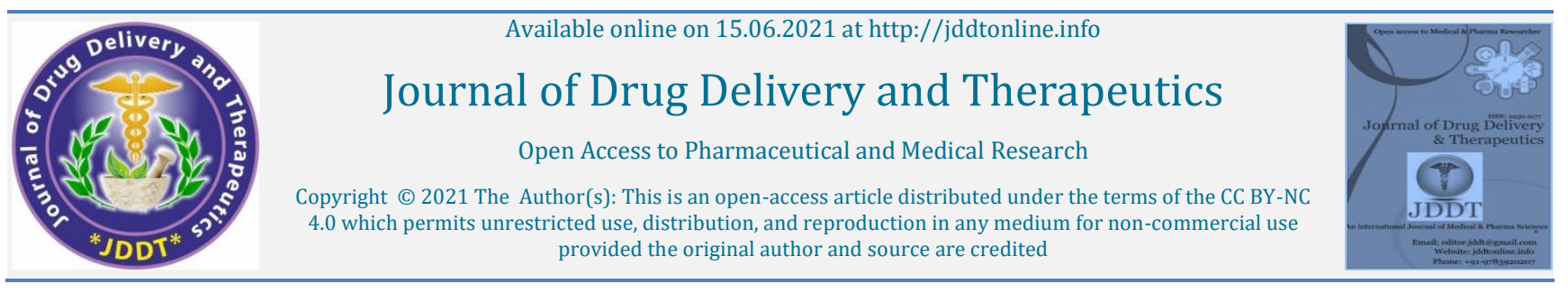

Access Full Text Article

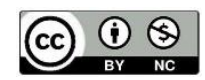

Review Article

\title{
In Silico Studies Comparing the Adjuvant Therapies Approved for Parkinson's disease
}

\section{(DSathish Kumar Jayaprakash*}

SRM College of Pharmacy, SRM IST, Kattankulathur, Kancheepuram, Tamil Nadu, India

\begin{tabular}{|c|c|}
\hline \multicolumn{2}{|l|}{ Article Info: } \\
\hline 回被回 & $\begin{array}{l}\text { Article History: } \\
\text { Received } 11 \text { April } 2021 \\
\text { Review Completed } 19 \text { May } 2021 \\
\text { Accepted } 23 \text { May } 2021 \\
\text { Available online } 15 \text { June } 2021\end{array}$ \\
\hline \multicolumn{2}{|c|}{ Cite this article as: } \\
\hline \multicolumn{2}{|c|}{$\begin{array}{l}\text { Sathish Kumar J, In Silico Studies Comparing the } \\
\text { Adjuvant Therapies Approved for Parkinson's } \\
\text { disease, Journal of Drug Delivery and Therapeutics. } \\
\text { 2021; } 11(3-\text { S):104-110 } \\
\text { D0I: http://dx.doi.org/10.22270/jddt.v11i3-S.4813 }\end{array}$} \\
\hline \multicolumn{2}{|c|}{ *Address for Correspondence: } \\
\hline $\begin{array}{l}\text { Sathish Kumar } \\
\text { Pharmacy, SRM } \\
\text { Tamil Nadu, Indi } \\
\text { 0002-6353-0960 }\end{array}$ & $\begin{array}{l}\text { Jayaprakash, SRM College of } \\
\text { T, Kattankulathur, Kancheepuram, } \\
\text { ORCID ID: https://orcid.org/0000 } \\
\text { Mail ID: sachinezio@gmail.com }\end{array}$ \\
\hline
\end{tabular}

\begin{abstract}
One of the most common neurodegenerative disorders is Parkinson's disease. The occurrence of Parkinson's disease includes loss of dopaminergic function and loss of motor function. It is characterized by non- motor as well as motor features. The medicine sector has been primarily focusing on developing adjunctive therapy for the treatment of Parkinson's disease in order to improve the efficacy of the drugs and to improve the quality of treatment. This study aims in silico screening of the drugs available for adjunctive therapy of Parkinson's disease. The pharmacokinetic parameters, potential adverse effects, toxicity studies, biological activity and the structure activity relationships of the drugs were analyzed and summarized. The drugs chosen as adjunctive therapy in the treatment of Parkinson's disease have reported high chances of adverse drug events and require precautions before administration. Although, they are therapeutically effective it is necessary to investigate other available therapies and then conclude their efficacy.
\end{abstract}

Keywords: Adjunct therapy, in silico, Parkinson's disease, pharmacokinetic study, toxicity profile.

\section{INTRODUCTION:}

One of the most common neurodegenerative disorders is Parkinson's disease. The occurrence of Parkinson's disease includes loss of dopaminergic function and loss of motor function. It is characterized by non- motor as well as motor features. It induces degenerative effects on control over mobility and muscle coordination. Non motor presentations of Parkinson's disease include disturbances in sleep cycle, cognitive changes and episodes of depression. The prevalence is higher in males to females with a ratio of $3: 2$. The delayed onset of the disease in females is associated with high estrogen as it works as a neuroprotective agent on the nigrostriatal dopaminergic system. Parkinson's disease at the end stage may lead to severe complications such as pneumonia and may even result in fatality. Some of the most common risk factors are environmental toxins, formation of free radicals and oxidative stress. However, genetic mutations are also associated with the condition. The main aim of the available treatment is to manage the symptoms and also prevent the progression of the disease 1 .

The drug therapy for Parkinson's disease includes the drugs or compounds that regulate the dopamine levels in brain thereby, reducing the severity of the disease and preventing its progression. The most commonly prescribed drug and the first line drug for the treatment of Parkinson's disease is
Levodopa. Levodopa belongs to a class of drugs called DOPA decarboxylase inhibitor. Levodopa, on administration enter the brain and is converted to dopamine. Other drugs used are monoamine oxidase- B inhibitors, COMT inhibitors and dopamine agonists. For the management of motor functions, drugs belonging to class, anticholinergics and antispasmodics are used. For treating the non- motor symptoms, droxidopa (for hypotension) and pimavanserin (for psychosis) was approved by FDA 2.

The medicine sector has been primarily focusing on developing adjunctive therapy for the treatment of Parkinson's disease. Adjunctive therapy refers to the addition of drugs or treatment methods to existing medication practice in order to improve the efficacy of the drugs and to improve the quality of treatment. Many drugs have been chosen for analysis to check if they are suitable for adjunctive therapy in the management of Parkinson's disease. FDA has approved a few drugs in the past few years and they have proved to be highly effective in the management of the disease. These days the drugs are available in combinations and are highly efficacious.

Safinamide is one of the drugs that have been recently approved for the treatment of Parkinson's disease. Being a benzyl amino derivative, it has numerous characters like 
anti-convulsant property, antiparkinsonian effects and neuroprotective features 3 .

Citicoline is a precursor of phospholipid synthesis and it is a source for choline in the metabolic pathway of acetylcholine biosynthesis in the body. It is known to improve the metabolism in cerebrum and also has neuroprotective properties. It has been inferred from the studies earlier conducted that citicoline is effective in treating Parkinsonism and also provides great aid in managing levodopa- induced psychoses 4 .

Opicapone is a COMT inhibitor that is taken along with levodopa to enhance its effect for improved symptom control. It is also known to prevent the breakdown of levodopa thereby, ensuring maximum efficacy ${ }^{5}$. D- serine has shown to improve the negative symptoms exhibited by schizophrenic patients as a result of anti- psychotic druginduced Parkinsonian symptoms. The study suggests that Dserine may be beneficial in the treatment of Parkinson's disease $^{6}$. Istradefylline is an adenosine A2A antagonist that is newly developed for the treatment of Parkinsonism. It has been found to be effective as it has potentially low side effects and it reduces OFF time when used in combination with levodopa. This was concluded from a study conducted among the different adjunctive therapies in combination with levodopa ${ }^{7}$.

This study aims in silico screening of the drugs available for adjunctive therapy of Parkinson's disease. The pharmacokinetic parameters, potential adverse effects, toxicity studies, biological activity and the structure activity relationships of the drugs were analysed and summarized. Various online servers and web resources were employed in this study. The use of online and offline tools for the prediction and evaluation of the various drug properties and parameters have led to evidential conclusion of the study. Also, the evidential data might provide great help in carrying out further studies related to this topic.

\section{METHODOLOGY:}

\subsection{Collection of data:}

The disease was studied in detail and the approved treatment and medication was inferred from the data published by American Parkinson Disease Association( APDA) ${ }^{8}$ The list of drugs approved for the treatment of Parkinson's disease was collected and their mechanism of action and other properties were studied.

\subsection{Pharmacokinetic Assessment:}

The ADMET parameters of the chosen drugs were assessed using the pKCSM online server9, 10,11,12. Various properties like the factors concerning the absorption, distribution, metabolism, excretion and toxicity were collected from the server. It is essential to calculate the pharmacokinetic properties in order to study and analyse the function of drugs inside the body. The admetSAR online server was also used for the assessment of the pharmacokinetic properties. The end points in the modeling of the selected drugs are

- LD50 single point estimates

- Very toxic binary classification

- Non- toxic binary classification

- EPA's 4- category hazard classification

- GHS 5- category hazard classification 13

2.3. In silico prediction for toxicity:
The toxicity of the targeted drugs was studied using OSIRIS® Property Explorer program ${ }^{14}$. This program is made accessible via cheminformatics.ch and chemistry. The tool enables one to analyse the mutagenicity, tumorigenicity, reproductive effects and irritant effects of a particular drug. The effects are indicated in green, yellow and red colours that represent drug conform behaviour, medium risk and high risk for mutagenicity or low absorption in intestine respectively. The method is highly useful in eliminating harmful drugs in advance during the discovery of drugs and in their development. The tool also provides access to the drug score, T. P. S. A and drug likeness.

i. T. P. S. A- The penetration across blood- brain barrier and the absorption of drugs in intestine are properties associated with bioavailability of the drugs. The properties are correlated with T. P. S. A and is calculated as the sum of the fragment contribution mainly, $\mathrm{O}$ - and $\mathrm{N}$ - fragments.

ii. Drug likeness- The drug likeness was also generated using OSIRIS program. Positive value as a result for drug likeness indicates that the compounds involved in the study are the fragments that are found in formulations that are commercially available.

iii. Overall drug score- A drug is considered favorable if the score is $>0.5$ and if the drug has minimal toxicity.

\subsection{Toxicity Predictions:}

Toxicity profile of the drugs was calculated from the T. E. S. T (Toxicity estimation software tool) ® Version 5.1. Histidine kinase is present in the system which will interact with the extracellular signal by phosphorylating the cytoplasmic receptor regulator.

The calculation was carried out using the consensus method with three end points. The toxicity of the drugs was calculated with Fathead minnow LC50(96 hr), Daphnia magna LC50(48 hr) and Bioconcentration factor as end points.

\subsection{Molecular Property Assessment:}

In silico screening of the selected drugs were performed using MOLINSPIRATION ${ }^{\circledR}$ software to evaluate the drug likeness and to predict their bioactivity. It also helps to screen the fragments present in the drugs. The drugs with a score of 0.00 for bioactivity is considered to be ideal biological activity, bioactivity score ranging from -0.50- 0.00 indicates moderate activity and $<0.00$ means, the drug is inactive.

\subsection{In silico Toxicity Prediction Using ProTox- II:}

The drugs were estimated for various end points. The models used in prediction are built of the data available from both in vivo and in vitro assay. The end points studied were cytotoxicity, hepatotoxicity, carcinogenicity, immunotoxicity, adverse effects and other targets for toxicity. This model for toxicity prediction incorporates knowledge from varied fields like biostatistics, toxicology, system biology etc ${ }^{15}$.

\subsection{Prediction for biological activity using PASS online:}

The Pass online web resource was used to predict the biological activity of the drugs. The biological activity spectrum of the drugs is predicted on the basis of the structural formula of the drugs. The analysis is purely based on the structure- activity relationship ${ }^{16}$.The Prediction of Activity Spectra for Substances (PASS) was carries out for the selected drugs. The biological activities result from interaction of the compounds present in the drugs and the 
biological objects. The activity of drug is dependent upon its activity and therefore SAR of the drug should be estimated for its pharmacological effects ${ }^{17}$. The adverse effects of the drugs were also predicted using PASS ONLINE web resource.

\section{RESULT AND DISCUSSION:}

\subsection{Drug likeness and toxicity prediction:}

The OSIRIS property explorer was used to determine the physicochemical properties of the five selected drug compounds. Citicoline and Opicapone show higher TPSA values $(233.12,150.66)$. The absorption, transport and penetration are based on the TPSA value of the drugs 18 None of the drugs indicate mutagenicity, irritancy, Tumorigenicity or reproductive effect considering the results obtained from OSIRIS property explorer. The drug likeness value of Opicapone was comparatively lower than the other four compounds (-3.54). A positive value indicates that the other four drugs contain fragments that are mostly present in the commercial drugs available in the market. The drug score is the summation of the cLogP, logs, molecular weight and toxicity risks. The purpose of drug score is to evaluate the potential of a chemical compound to meet the criteria of a possible drug candidate. The drug score of Citicoline $(0.38)$ is less than the other four compounds ${ }^{19}$. The results obtained from OSIRIS are listed in table 5.

\subsection{Evaluation of pharmacokinetic profile using admetSAR:}

AdmetSAR was used to study the absorption, distribution, metabolism, excretion and toxicity profile of the selected drugs belonging to adjunct therapy. Citicoline, Istradefylline, Opicapone and Safinamide showed excellent human intestinal absorption. Absorption of the drug plays a major role in achieving the systemic effect of the drug. Post oral administration, the drug has to resist the effect of many enzymes and pH environments. And then is absorbed in the intestinal layer which has hindrance of many barriers formed by the cell membrane 20 .

Istradefylline is positive for Caco-2 assay. The human colon epithelial cancer cells mimic the function of human intestinal epithelium. This parameter indicates good permeability of the drug across the intestinal epithelium ${ }^{21}$.

We can treat Parkinson's disease more effectively when a drug passes the Blood Brain Barrier by achieving satisfactory bioavailability and good anti- parkinsonian efficacy. All the drugs except D-Serine pass the Blood Brain Barrier. Except D-Serine all other drug shows Blood Brain Barrier permeability 22 .

Human oral bioavailability is important for a drug to bring its therapeutic effect into action. Poor oral drug availability of a drug may lead to metabolism and elimination without reaching the systemic blood circulation ${ }^{23}$. The selected drugs except Citicoline show great human oral bioavailability.

A protein called $\alpha$-synuclein containing140 amino acids disturbs the normal function of intra-cellular targets. Recent studies report that $\alpha$-synuclein binds to mitochondria in substantia nigra and cortex of Parkinson's disease brain. This may lead to deregulation of calcium signal. Deregulation of Calcium signal causes protein miss-folding, impaired metabolism and apoptosis. So, it is necessary for the drug to exert its therapeutic effect on mitochondrial membrane 24,25 . AdmetSAR shows that drugs except D-Serine exhibits mitochondrial localization.

The five selected drugs show no sign of carcinogenicity or eye corrosion. D-Serine is positive for eye irritation.
Citicoline is under class 4 acute oral toxicity and the other four drugs are under class 3.

The solubility of a drug in an aqueous medium affects its absorption and distribution characteristics. Poor solubility of a drug may result in bad absorption and distribution. This leads to gastrointestinal toxicity and may affect the bioavailability of a drug ${ }^{26}$. Range of water solubility in mol/L is mentioned in Table1 ${ }^{27}$. Safinamide shows very less water solubility value compared to other selected drugs (-3.572).

Plasma protein binding is one of the most important factors in studying the activity of a drug. The plasma protein's function is to control the free drug concentration. High concentration of free drug may pave way to severe toxicity 28. D-serine has the lowest value for PPB $(-0.11)$ and Istradefylline has the highest value (0.942).

Micronucleus assay is used to study the potential of a drug to cause genotoxicity and mutation in the human genome. All five compounds are positive for micronucleus assay 29 . The pharmacokinetic parameters obtained from admetSAR are given in Table 6.

\subsection{Estimation of Pharmacokinetic profile using pkCSM:}

All the five drugs $(-2.474,-2.888,-2.934,-3.175,-2.483)$ are slightly soluble in aqueous medium. Poor water solubility indicates poor absorption and distribution of the drug. Caco2 permeability is measured in log Papp in $10^{-6} \mathrm{~cm} / \mathrm{s}$. The Caco 2 normal range is listed in table 2 . All the five compounds show low Caco2 permeability $(0.498,0.441,1.485,0.322$, and 0.794). Istradefylline, Opicapone, Safinamide and D-Serine have high human intestinal absorption rate (99.278, 80.836, 94.015 and 68.865). Citicoline has low human intestinal absorption rate (27.479). The normal range for HIA\% is listed in table 3. Parameters generated from pkCSM are given in Table 7.

\subsection{Prediction of toxicity using ProTox II:}

Amount of dose that causes $50 \%$ death in the subject is called LD50 of a drug. The LD50 test is used to assess the potential hazardous effect caused by the drug and to screen the level safety ${ }^{33}$. Citicoline is under class VI, therefore it is completely safe $(12000 \mathrm{mg} / \mathrm{kg})$ (Table 4). Istradefylline $(19 \mathrm{mg} / \mathrm{kg})$ comes under class II toxicity indicating high chances of fatality.

All the selected drugs follow Lipinski's rule where the molecular weight does not exceed 500 Dalton. Log P value must be less than 5 , number of hydrogen bond donor must be less than 5 . Molar refractivity value must be 40-130 and the number hydrogen bond acceptor must be less than 1034 . Results generated from ProTox II are given in Table 8.

\subsection{Prediction of bioactivity score using Molinspirstion:}

The bioactivity score of the drug is necessary to study the pharmacological effect. The drugs bind to certain receptors, enzymes and ion channels to exert their action. These sites are called biological targets. The drugs' bioactivity score was calculated for the drug binding to G-Protein Coupled Receptor, Ligand, kinase inhibitor, nuclear receptor Ligand, protease inhibitor, enzyme inhibitor and ion channel modulator ${ }^{35}$. A drug with bioactivity scores more than 0.00 is considered to have biological activities, score -5.0 to 0.00 will have moderate activity and score less than 0.00 is considered inactive ${ }^{36}$.

Citicoline (1.31) shows better binding to G-Protein coupled receptor Ligand, protease inhibitor, kinase inhibitor and 
enzyme inhibitor compared to other drugs. Bioactivity score of the drugs is listed in Table 9.

\subsection{Prediction of possible ADR and side effects using PASS online:}

This table contains probability of both $\mathrm{Pa}$ and $\mathrm{Pi}$. $\mathrm{Pa}$ is the probability of belonging to "actives" and $\mathrm{Pi}$ is the probability of the drug belonging to the "inactives"14. Here we have listed the drug with Pa>Pi. Results are interpreted in Table 10. PD patient are observed with psychomotor dysfunction characterized by behavioural changes, sensory disturbances, sleep disturbance, depression, alteration in consciousness. In order to check if there are any ADR of the drug which may result in aggravating the disease, we have used PASS online.

\section{CONCLUSION:}

Parkinson's disease is neurodegenerative disease which affects the dopamine system and motor activity. Parkinson's disease has no permanent cure but it can be treated symptomatically to improve the patient's quality of life. The patients are treated with dopamine agonist, MAO inhibitor, and COMT inhibitor in order to increase the level of dopamine and to improve psychomotor activity. Adjunct therapy is also given to improve therapeutic efficacy. Although drugs are approved for the treatment of Parkinson's disease and many other drugs are under investigation and trials, all the properties should be properly assessed before the use of these drugs. The toxicity profile as well as their efficacy should be studied. In an attempt to study the properties and the toxic effects of the drugs used in adjunct therapy, in silico screening was carried out in order to predict the interactions of the drugs. However, it is not a definite conclusion if the drugs when used as adjunct therapy, alleviate the interactions or not. There are high chance of occurrence of adverse drug events and toxicity, we should monitor the patient for symptoms and must consider dose adjustment if necessary. However, with further experimentation using the results of this study will help us to identify the combination of drugs that yield maximum therapeutic effects and lesser complications. Further studies are necessary to understand more about the pharmacokinetic profile, side effects and toxicity of the drugs that can be used as adjunct therapy in Parkinson's disease. Studies and clinical trials for use of alternative therapy must be considered.

\section{DECLARATIONS}

Ethics approval and consent to participate

Not applicable.

Consent for publication

Not applicable.

Availability of data and material

All data and material are available upon request.

Competing interests

The authors declare no conflict of interest in the contents of the manuscript.

Funding

No funding received.

Table1. Range of solubility

\begin{tabular}{ll}
\hline Value of Water Solubility $(\log S)$ & Solubility \\
\hline More than 0 & Highly soluble \\
$\mathbf{0}$ to -2 & Moderately soluble \\
$\mathbf{- 2}$ to -4 & Slightly soluble \\
Less than -4 & Insoluble \\
\hline
\end{tabular}

Table 2. Range of Caco2 permeability value. ${ }^{30}$

\begin{tabular}{lll}
\hline Caco2 assay value & Range & Percentage \\
\hline Papp $\leq 10-6$ cm/s & Low & $0-20 \%$ \\
$\mathbf{1 0 - 6 c m} / \mathbf{s}<$ Papp $\leq 10 \times 10-6$ cm/s & Medium & $20-70 \%$ \\
Papp $>10 \times 10-6 ~ c m / s$ & High & $70-100 \%$ \\
\hline
\end{tabular}

Table 3. Range of human intestinal absorption in percentage 31

\begin{tabular}{ll}
\hline HIA in percentage & Absorption rate \\
\hline $100-67$ & High \\
$66-33$ & Moderate \\
$32-0$ & Low \\
\hline
\end{tabular}

Table 4. Classification of LD50 based on GHS32

\begin{tabular}{lll}
\hline LD50 $(\mathbf{m g} / \mathbf{k g})$ & Class & Result \\
\hline LD50 $\leq \mathbf{5}$ & I & Fatal \\
$\mathbf{5}<$ LD50 $\leq \mathbf{5 0}$ & II & Fatal \\
$\mathbf{5 0}<$ LD50 $\leq \mathbf{3 0 0}$ & III & Toxic \\
$\mathbf{3 0 0}<$ LD50 $\leq \mathbf{3 0 0}$ & IV & Harmful \\
$\mathbf{2 0 0 0}<$ LD50 $\leq \mathbf{5 0 0 0}$ & V & Maybe harmful \\
$\mathbf{L D 5 0}>\mathbf{5 0 0 0}$ & VI & Nontoxic \\
\hline
\end{tabular}


Table 5. Estimation of Toxicity profile of drugs used in adjunct therapy Using Osiris

\begin{tabular}{llllll}
\hline Parameters & Opicapone & Safinamide & Istradefylline & Citicoline & D- Serine \\
\hline Mutagenic & GREEN & GREEN & GREEN & GREEN & GREEN \\
Tumorigenic & GREEN & GREEN & GREEN & GREEN & GREEN \\
\hline Irritant & GREEN & GREEN & GREEN & GREEN & GREEN \\
Reproductive effect & GREEN & GREEN & GREEN & GREEN & GREEN \\
TPSA & 150.66 & 64.35 & 76.90 & 233.12 & 83.55 \\
Drug likeness & -3.54 & 0.55 & 7.34 & 51.38 & -10.07 \\
Drug score & 0.43 & 0.77 & 0.86 & 0.38 & 0.50 \\
\hline
\end{tabular}

Table 6. Estimation of pharmacokinetic profile of drugs used in adjunct therapy using ADMET SAR

\begin{tabular}{llllll}
\hline ADMET & Citicoline & D-Serine & Istradefylline & Opicapone & Safinamide \\
\hline Water solubility (logS) & -2.735 & 0.175 & -2.902 & -3.285 & -3.572 \\
Plasma protein binding (100\%) & 0.835 & 0.11 & 0.942 & 0.807 & 0.795 \\
Acute oral toxicity(kg/mol) & 3.868 & 0.328 & 2.428 & 2.741 & 2.662 \\
Human Intestinal Absorption & + & - & + & + & + \\
Caco-2 & - & - & + & - & - \\
Blood Brain Barrier & + & - & + & + & + \\
Human oral bioavailability & - & + & Mitochondria & Mitochondria & Mitochondria \\
Subcellular localization & Mitochondria & Lysosomes & - & - & - \\
Carcinogenicity (binary) & - & - & - & - & - \\
Eye corrosion & - & - & - & - & - \\
Eye irritation & - & + & + & + & - \\
Micronucleus assay & + & + & + & III & III \\
Hepatotoxicity & - & - & & III \\
Acute Oral Toxicity (c) & IV & III & & & + \\
\hline
\end{tabular}

Table 7. Estimation of pharmacokinetic parameter of drugs used in adjunct therapy using pkCSM.

\begin{tabular}{llllll}
\hline PARAMETER & Citicoline & D-Serine & Istradefylline & Opicapone & Safinamide \\
\hline Water solubility (log mol/L) & -2.474 & -2.888 & -2.954 & -3.175 & -2.483 \\
CaCo2 permeability (log Papp in 10-6 cm/s) & 0.498 & 0.441 & 1.485 & 0.322 & 0.794 \\
Intestinal absorption (\% Absorbed) & 27.479 & 68.865 & 99.278 & 80.836 & 94.015 \\
BBB Permeability (log BB) & -1.873 & -0.709 & -1.358 & -1.369 & -0.388 \\
Oral route Acute Toxicity (LD50) (mol/kg) & 1.905 & 1.967 & 2.501 & 2.905 & 1.989 \\
Oral route chronic toxicity (log mg/kg_bw/day) & 2.377 & 2.971 & 0.707 & 1.717 & 1.835 \\
Hepatotoxicity & Yes & No & Yes & Yes & Yes \\
\hline
\end{tabular}

Table 8: Prediction of Toxicity of drugs used in adjunct therapy Using ProTox II

\begin{tabular}{llllll}
\hline Parameter & Opicapone & Safinamide & Istradefylline & Citicoline & D- Serine \\
\hline Predicted LD50(Mg/Kg) & 1000 & 2000 & 19 & 12000 & 2000 \\
Toxicity Class & 4 & 4 & 2 & 6 & 2 \\
Average Similarity (\%) & 38.01 & 57.18 & 60.99 & 36.27 & 70.28 \\
Molecular Weight & 413.17 & 302.34 & 384.43 & 478.25 & 105.09 \\
$\begin{array}{l}\text { No Of Hydrogen Bond } \\
\text { Acceptor }\end{array}$ & 15 & 23 & 29 & 29 & 11 \\
No of Hydrogen Bond & 2 & & & 1 & 3 \\
Donor & & & & 47 & 14 \\
No of Atoms & 37 & 41 & 52 & 48 & 13 \\
No of Bonds & 39 & 42 & 54 & 10 & 2 \\
No Of Rotatable Bond & 3 & 7 & 6 & 99.61 & 22.18 \\
Molecular Refractivity & 99.15 & 82.48 & 110.01 & 166.64 & 83.55 \\
TPSA & 150.66 & 64.35 & 80.28 & -0.25 & -0.19 \\
Water Partition & 4.6 & 3.46 & 2.12 & & \\
Coeffecient (Logp) & & & & & \\
\hline
\end{tabular}


Table 9. prediction of bioactivity score using molinspiration

\begin{tabular}{llllll}
\hline PARAMETERS & Opicapone & Safinamide & Istradefylline & Citicoline & D- serine \\
\hline GPCR Ligand & -0.27 & 0.18 & 0.04 & 1.31 & -2.66 \\
Ion channel modulator & -0.28 & -0.04 & -0.55 & 0.86 & -2.54 \\
Kinase inhibitor & -0.41 & -0.01 & -0.53 & 0.67 & -3.34 \\
Nuclear receptor ligand & -0.34 & -0.18 & -0.95 & -0.83 & -3.34 \\
Protease inhibitor & -0.39 & 0.29 & -0.66 & 0.49 & -2.36 \\
Enzyme inhibitor & -0.14 & 0.04 & 0.00 & 1.54 & -2.38 \\
\hline
\end{tabular}

Table 10. Prediction of ADR and side effects of drugs used in adjunct therapy using PASS online.

\begin{tabular}{|c|c|c|c|c|c|c|c|c|c|c|}
\hline \multirow[t]{2}{*}{ ADR/SIDE EFFECT } & \multicolumn{2}{|c|}{ CITICOLINE } & \multicolumn{2}{|c|}{ D-SERINE } & \multicolumn{2}{|c|}{ ISTRADEFYLLINE } & \multicolumn{2}{|c|}{ OPICAPONE } & \multicolumn{2}{|c|}{ SAFINAMIDE } \\
\hline & $\mathrm{Pa}$ & $\mathrm{Pi}$ & $\mathrm{Pa}$ & $\mathrm{Pa}$ & $\mathrm{Pi}$ & $\mathrm{Pi}$ & $\mathrm{Pa}$ & $\mathrm{Pi}$ & $\mathrm{Pa}$ & $\mathrm{Pi}$ \\
\hline Anaemia & 0.985 & 0.003 & 0.909 & -- & -- & 0.002 & -- & -- & -- & -- \\
\hline Neurotoxicity & 0.977 & 0.002 & 0.743 & -- & -- & 0.024 & -- & -- & -- & -- \\
\hline Sleep disturbance & 0.917 & 0.009 & 0.609 & 0.292 & 0.211 & 0.059 & -- & -- & -- & -- \\
\hline Sensory disturbance & 0.902 & 0.009 & 0.610 & -- & -- & 0.059 & -- & -- & -- & -- \\
\hline Conscious alteration & 0.799 & 0.019 & 0.629 & -- & -- & 0.043 & -- & -- & -- & -- \\
\hline Ocular toxicity & 0.782 & 0.025 & 0.669 & -- & -- & 0.041 & -- & -- & -- & -- \\
\hline $\begin{array}{l}\text { Behavioural } \\
\text { disturbance }\end{array}$ & 0.830 & 0.020 & 0.595 & 0.281 & 0.196 & 0.057 & -- & -- & -- & -- \\
\hline Hypnotic & -- & -- & -- & -- & -- & -- & 0.186 & 0.101 & -- & -- \\
\hline Withdrawal & -- & -- & -- & -- & -- & -- & -- & -- & 0.356 & 0.202 \\
\hline $\begin{array}{l}\text { Psychomotor } \\
\text { impairment }\end{array}$ & -- & -- & 0.577 & 0.061 & 0.250 & 0.241 & -- & -- & 0.269 & 1.219 \\
\hline Muscle weakness & 0.240 & 0.201 & 0.736 & 0.019 & -- & -- & 0.224 & 0.211 & -- & -- \\
\hline Urine discolouration & -- & -- & 0.881 & 0.004 & 0.196 & 0.113 & 0.179 & 0.127 & 0.227 & 0.094 \\
\hline
\end{tabular}

Table 11. Estimated toxicity profile of drugs used in adjunct therapy with T.E.S.T.

\begin{tabular}{|c|c|c|c|c|c|}
\hline Parameters & Opicapone & Safinamide & Istradefylline & Citicoline & D-Serine \\
\hline $\begin{array}{l}\text { Fathead minnow LC50 (96 hr) - } \\
\text { Log10(mol/L) }\end{array}$ & $\mathbf{N} / \mathbf{A}$ & 4.96 & $\mathbf{N} / \mathbf{A}$ & N/A & 1.73 \\
\hline Fathead minnow LC $50(96 \mathrm{hr}) \mathrm{mg} / \mathrm{L}$ & $\mathbf{N} / \mathbf{A}$ & 3.28 & $\mathbf{N} / \mathbf{A}$ & N/A & 1935.54 \\
\hline $\begin{array}{l}\text { Similarity coefficient } \\
(\geq 0.5)\end{array}$ & 0.77 & 0.89 & 0.50 & 0.28 & 0.60 \\
\hline $\begin{array}{l}\text { Daphnia magna LC } 50(48 \mathrm{hr}) \text { - } \\
\log 10(\mathrm{~mol} / \mathrm{L})\end{array}$ & $\mathbf{N} / \mathbf{A}$ & 5.39 & 4.32 & N/A & 2.52 \\
\hline Daphnia magna LC50 (48 hr) mg/L & $\mathbf{N} / \mathbf{A}$ & 1.23 & 18.20 & N/A & 315.41 \\
\hline $\begin{array}{l}\text { Similarity coefficient } \\
(\geq 0.5)\end{array}$ & 0.77 & 0.93 & 0.33 & N/A & 0.67 \\
\hline Bioconcentration factor $\log 10$ & N/A & 1.23 & 1.37 & $\mathbf{N} / \mathbf{A}$ & -0.64 \\
\hline Bioconcentration factor & $\mathbf{N} / \mathbf{A}$ & 16.87 & 23.23 & N/A & 0.23 \\
\hline $\begin{array}{l}\text { Similarity coefficient } \\
(\geq 0.5)\end{array}$ & 0.78 & 0.82 & 0.71 & 0.96 & 0.24 \\
\hline
\end{tabular}

Similarity coefficient $(\geq 0.5$ ) comparing the category of similar structures form the existing compounds. 


\section{REFERENCES:}

1. DeMaagd G, Philip A, "Parkinson's Disease and Its Management: Part 1: Disease Entity, Risk Factors, Pathophysiology, Clinical Presentation, and Diagnosis" P T, 2015; 40(8):504-32.

2. Parkinson's Disease. Parkinson's News Today; 2021 [cited 18 May 2021]. Available at:

https://parkinsonsnewstoday.com/parkinsons-diseasetreatments/. Accessed May 18, 2021.

3. Stocchi F, Torti M, "Adjuvant Therapies For Parkinson's Disease: Critical Evaluation of Safinamide" Drug Des Devel Ther, 2016; 10:609-618 https://doi.org/10.2147/DDDT.S77749

4. Que D-LS, Jamora RDG, "Citicoline as Adjuvant Therapy in Parkinson's Disease: A Systematic Review" Clin. Ther, 2021; 43(1):e19-e31. https://doi.org/10.1016/j.clinthera.2020.11.009

5. Newly Approved Parkinson's Drug Aims to Lessen "Off" Time. The Michael J. Fox Foundation for Parkinson's Research. Parkinson's Disease; 2021. Available at:

https://www.michaeljfox.org/news/newly-approvedparkinsons-drug-aims-lessen-time. Accessed May18, 2021

6. Gelfin E, Kaufman Y, Korn-Lubetzki I, Bloch B, Kremer I, Javitt DC, et al, "D-Serine Adjuvant Treatment Alleviates Behavioural and Motor Symptoms in Parkinson's Disease" Int ] Neuropsychopharmacol, 2012; 15(4):543-9.

https://doi.org/10.1017/S1461145711001015

7. Torti M, Vacca L, Stocchi F, "Istradefylline for the treatment of Parkinson's disease: is it a promising strategy?" Expert Opin Pharmacother, 2018; 19(16):1821-1828 https://doi.org/10.1080/14656566.2018.1524876

8. Approved Medications, American Parkinson Disease Assoc. APDA; 2021. Available at: https://www.apdaparkinson.org/what-isparkinsons/treatment-medication/medication/. Accessed May 18, 2021.

9. Dhage MS, Nila G, Dhivya D, Sarvesh S, "In Silico Prediction and Pharmacokinetic Comparison of Ursodeoxycholic Acid and Obeticholic Acid in the Management of Primary Biliary Cholangitis" J. drug deliv. Ther, 2021; 11(2):113-117. https://doi.org/10.22270/jddt.v11i2-S.4669

10. Sarvesh S, Sathish kumar RC, Vijayakumar TM, "CYP3A4 Mediated Pharmacokinetic Drug interaction potential of MahaYogaraj Gugglu and E,Z Guggulsterone" Nature Res, 2021; 11(1):715. https://doi.org/10.1038/s41598-020-80595-5

11. Sarvesh S, Haripritha M, Preethi L, Arjun Gokulan M, Dhivya D, Nila G, Rahul R, "HCIP: an online database for prediction CYP450 Enzyme Inhibition Potential of Bioactive Compounds" J. drug deliv. Ther, 2021; 11(2). https://doi.org/10.22270/jddt.v11i2.4637

12. Sarvesh S, Vijayakumar TM, "Isomers of Guggulsterone in hyperlipidemia" Obs Med, 2021; 22(7,72):100326. https://doi.org/10.1016/j.obmed.2021.100326

13. Gadaleta D, Vuković K, Toma C, Lavado GJ, Karmaus AL, Mansouri $\mathrm{K}$, et al, "SAR and QSAR modeling of a large collection of LD50 rat acute oral toxicity data" J. Cheminformatics, 2019; 11(1):58. https://doi.org/10.1186/s13321-019-0383-2

14. Preethi L, Nila G, Dhivya D, Sarvesh S, "Therapeutic use of Guggulsterone in COVID-19 induced obesity (COVIBESITY) and significant role in immunomodulatory effect" ObesMed, 2021; 24:100346. https://doi.org/10.1016/j.obmed.2021.100346

15. Banerjee P, Eckert AO, Schrey AK, Preissner R, "ProTox-II: a Webserver for the Prediction of Toxicity of Chemicals" Nucleic Acids Res, 2018; 46(W1):W257-W63. https://doi.org/10.1093/nar/gky318

16. Filimonov DA, Lagunin AA, Gloriozova TA, Rudik AV, Druzhilovskii DS, Pogodin PV, et al, "Prediction of the Biological Activity Spectra of Organic Compounds Using the Pass Online Web Resource" Chem Heterocycl Comp,2014; 50(3):444-57. https://doi.org/10.1007/s10593-014-1496-1

17. Filimonov DA, Rudik AV, Dmitriev AV, Poroikov VV, "ComputerAided Estimation of Biological Activity Profiles of Drug-Like Compounds Taking into Account Their Metabolism in Human Body" Int. J. Mol. Sci, 2020; 21(20):7492. https://doi.org/10.3390/ijms21207492

18. Ertl P, Rohde B, Selzer P, "Fast Calculation of Molecular Polar Surface Area as a Sum of Fragment-Based Contributions and its Application to the Prediction of Drug Transport Properties" J. Med. Chem, 2000; 43(20):3714-3717. https://doi.org/10.1021/jm000942e
19. Ayati A, Falahati M, Irannejad H, Emami S,"Synthesis, In vitro Antifungal Evaluation and In silico Study of 3-azolyl-4chromanone phenylhydrazones" Daru, 2012; 20(1):46. https://doi.org/10.1186/2008-2231-20-46

20. Mannens GSJ, Bohets H, Verboven P, Steemans K, Lavrijsen K, Meuldermans W. Rapid Permeability Screening in Drug Discovery to Predict Human Intestinal Absorption. In: Pelkonen O., Baumann A., Reichel A. (eds) Pharmacokinetic Challenges in Drug Discovery. Ernst Schering Research Foundation Workshop, vol 37. Springer, Berlin, Heidelberg: 2002.p. 49-67. https://doi.org/10.1007/978-3-662-04383-7_3

21. van Breemen RB, Li Y, "Caco-2 Cell Permeability Assays to Measure Drug Absorption" Expert Opin Drug Metab Toxicol, 2005; 1(2):175-85 https://doi.org/10.1517/17425255.1.2.175

22. Xiong S, Li Z, Liu Y, Wang Q, Luo J, Chen X, et al, "Brain-targeted Delivery Shuttled by Black Phosphorus Nanostructure to Treat Parkinson's Disease" Biomaterials, 2020; 260:120339. https://doi.org/10.1016/j.biomaterials.2020.120339

23. Kim MT, Sedykh A, Chakravarti SK, Saiakhov RD, Zhu H,"Critical Evaluation of Human Oral Bioavailability for Pharmaceutical Drugs by using various Cheminformatics Approaches" Pharm Res, 2014; 31(4):1002-14. https://doi.org/10.1007/s11095013-1222-1

24. Guardia-Laguarta C, Area-Gomez E, Schon EA, Przedborski S, "Novel Subcellular Localization for $\alpha$-synuclein: Possible Functional Consequences" Front Neuroanat, 2015; 23(9):17. https://doi.org/10.3389/fnana.2015.00017

25. Stefanis L, " $\alpha$-Synuclein in Parkinson's disease" Cold Spring Harb Perspect Med, 2012; 2(2):a009399. https://doi.org/10.1101/cshperspect.a009399

26. Savjani KT, Gajjar AK, Savjani JK, "Drug Solubility: Importance and Enhancement Techniques" ISRN Pharm, 2012; 2012:195727. https://doi.org/10.5402/2012/195727

27. Sorkun MC, Khetan A, Er S, "AqSolDB, a Curated Reference Set of Aqueous Solubility and 2D Descriptors for a Diverse Set of Compounds" Sci Data, 2019; 6: 143. https://doi.org/10.1038/s41597-019-0151-1

28. Trainor GL, "The Importance of Plasma Protein Binding in Drug Discovery" Expert Opin Drug Discov, 2007; 2(1):51-64. https://doi.org/10.1517/17460441.2.1.51

29. Sommer S, Buraczewska I, Kruszewski M, "Micronucleus Assay: The State of Art, and Future Directions" Int J Mol Sci, 2020; 24;21(4):1534. https://doi.org/10.3390/ijms21041534

30. Caco-2 cells Permeability Assay Protocol - In vitro Intestinal Absorption Evaluation. ReadyCell In vitro Tools; 2021. Available at: https://readycell.com/caco-2-permeability-protocol/. Accessed May 18, 2021.

31. Damião M, Pasqualoto K, Polli M, Parise Filho R, "To Be Drug or Prodrug: Structure-Property Exploratory Approach Regarding Oral Bioavailability" J. Pharm. Pharm. Sci, 2014; 17:532-40. https://doi.org/10.18433/J3BS4H

32. Banerjee P, Eckert AO, Schrey AK, Preissner R, "ProTox-II: a webserver for the prediction of toxicity of chemicals" Nucleic Acids Res, 2018; 29, 46(W1):W257-W263. https://doi.org/10.1093/nar/gky318

33. Erhirhie EO, Ihekwereme CP, Ilodigwe EE, "Advances in Acute Toxicity Testing: Strengths, Weaknesses and Regulatory Acceptance" Interdiscip Toxicol, 2018; 11(1):5-12. https://doi.org/10.2478/intox-2018-0001

34. Lipinski CA, "Lead- and drug-like compounds: the rule-of-five revolution" Drug Discovery Today: Technologies, 2004; 1(4):337-41. https://doi.org/10.1016/j.ddtec.2004.11.007

35. Khan T, Dixit S, Ahmad R, Raza S, Azad I, Joshi S, Khan AR, "Molecular Docking, PASS Analysis, Bioactivity Score Prediction, Synthesis, Characterization and Biological Activity Evaluation of a Functionalized 2-butanone thiosemicarbazone Ligand and its Complexes" J Chem Biol. 2017; 10(3):91-104. https://doi.org/10.1007/s12154-017-0167-y

36. Husain A, Ahmad A, Khan S, Asif M, Bhutani R, Al-Abbasi F, "Synthesis, molecular properties, toxicity and biological evaluation of some new substituted Imidazolidine derivatives in search of potent Anti-inflammatory agents" Saudi Pharm J, 2015; 43. https://doi.org/10.1016/j.jsps.2015.02.008 